ENERGY TRANSPORT IN RADIO GALAXIES AND QUASARS:

\title{
A WORKSHOP THEORY REVIEW
}

\author{
PHILIP E. HARDEE \\ University of Alabama \\ Tuscaloosa, Alabama, 35487, USA
}

\begin{abstract}
A workshop on the dynamics, emission and morphology associated with the highly collimated outflows seen in radio galaxies and quasars was held at The University of Alabama in Tuscaloosa in September of 1995. Complete workshop proceedings will be published in the ASP Conference Series. This workshop review covers the theoretical developments and problems discussed at the workshop.
\end{abstract}

\section{Introduction}

It has been over ten years since the Green Bank workshop "Physics of Energy Transport in Extragalactic Radio Sources" was held to address the questions raised by detailed imaging of jets in active galaxies and quasars. In that workshop the focusing topics were (a) Jet Correlations and Observational Constraints, (b) Jet Sidedness, Velocity and Unification, (c) Confinement and Stability, and (d) Particle Acceleration, Entrainment, and Turbulence. Considerable work has occurred in the intervening decade. Much improved data at all wavelengths from radio to $\gamma$-rays are now available for these objects, and new families of unified models have been proposed to link classes of objects whose relationships were less clear a decade ago. The VLBA is coming on line and the latest supercomputers make it possible to perform three dimensional numerical experiments. Thus it seemed an auspicious time to review the progress made in the past decade, to evaluate the research being conducted in this area at the present time, and to address the issue of how best to utilize existing observational and computational resources to answer the questions that still remain concerning the highly collimated outflows associated with Radio Galaxies and Quasars. 


\section{Sub-Parsec and Parsec Scales: Acceleration, Collimation}

Energy transport begins with inward transport of fuel from the ISM, conventionally via an accretion disk around a black hole. Andrew Wilson argued that there is some evidence for powerful radio sources being triggered by merger events. The gas must be forced into the vicinity of the nuclear black hole in some special way conducive to the formation of jets, or in a different picture the merger events result in sufficient black hole rotational energy to power the jets. The reason or reasons for the difference in the active nature of spiral and elliptical galaxies, and of radio loud and radio quiet quasars remain an open question. On the other hand, theories of jet formation seem to be converging towards magnetic acceleration and collimation schemes, although Peter Scheuer cautioned that such convergence may be premature.

Magnetic acceleration and collimation schemes where the magnetic field is anchored in a Keplerian accretion disk seem particularly promising at the present time. In these schemes disk rotation twists an initial field which then becomes capable of accelerating a disk corona via magnetic and pressure gradient forces. Collimation is mainly the result of the pinching force associated with the toroidal magnetic field. While numerical experiments give promising results, the experiments are not yet fully self-consistent; e.g., in the work presented by Richard Lovelace the accretion disk is treated as a boundary condition on the electromagnetic fields without consideration of the back reaction on the disk. In addition to back reaction on the disk material, disk instabilities may play an important role in jet production schemes. Dimitris Christodoulou demonstrated the use of an energy variational principle to approach accretion disk instabilities in a way that allows the addition of a toroidal field to the poloidal field configuration assumed in previous stability work (e.g., Balbus \& Hawley 1991). This new work suggests an explosive release of an amplified toroidal field which might account for variation in jet speed. Future work will require incorporation of more realistic boundary conditions in the numerical simulations and will also require the investigation of magnetic fields, supported by external currents, that appear to thread a black hole's event horizon. Such schemes extract the rotational energy of a spinning black hole (e.g., Blandford 1992).

Magnetic collimation and acceleration implies strong toroidal magnetic fields and significant jet axial current flows. Thus, current driven instability as opposed to dynamically driven Kelvin-Helmholtz instability (e.g., Birkinshaw 1991) of the jet may be important. Steffan Appl presented results showing that current driven modes have growth rates significantly less than the Kelvin-Helmholtz modes for cases of astrophysical interest. If strong magnetic fields exist in the medium external to the jet then magnetic 
bending can lead to distorted flow structures. Several numerical simulations presented by Shinji Koide demonstrate that a jet trans-Alfvènic relative to a strong external magnetic field can be redirected. Thus, magnetic bending provides a viable option to jet cloud collisions as a jet deflection mechanism where magnetic fields in the external environment are large.

\section{Relativistic Jets: Simulation, Modeling, Emission}

A leaning towards relativistic jet production was evident at the workshop and relativistic numerical simulation results from three different groups (P. Hughes \& C. Duncan; S. Komissarov \& S. Falle; J. Marti \& J. Gomez) were presented. At the present time all work remains axisymmetric and an obvious next step is to proceed towards fully three dimensional simulations. Probably the most significant difference between relativistic and non-relativistic jet dynamics lies in the regime where the jet's sound speed is high relative to the flow speed. While non-relativistic transonic jets are very unstable the relativistic jet can be stabilized by a high Lorentz factor. Other differences are more subtle and occur because of the different relationship between energy and momentum fluxes in non-relativistic and relativistic flows. It will be important to quantify the differences between non-relativistic and relativistic flows. One of the important aspects of relativistic jet simulations will be to provide a firm dynamical basis for the calculation of radiation beamed and boosted by the relativistic flow, and from shocks residing within the flow. Both single and multiple shock structures and emission were presented at the workshop and Sergeui Komissarov showed results where the shock structures were moving relativistically. Not surprisingly the appearance of the jets containing such structures proved very dependent on the viewing angle.

The present observational and theoretical situation reviewed by Alan Marscher suggests that theoretical efforts need to focus on the synchrotron processes associated with accelerating, expanding relativistic jets which may contain relativistically moving shocks with Lorentz factor different from that of the relativistically moving outflow. In this regard Markos Georganopoulos presented a detailed calculation of the synchrotron radiation coming from a relativistically moving feature for which amplitudes and variability decrease with frequency and with viewing angle. Such a result is suggestive of the variability difference between $\mathrm{X}$-ray (lesser variability) and radio (greater variability) selected BL Lacs. Future progress in this area depends upon model building and observations involving multi-frequency source monitoring at high frequencies capable of probing the jet, interior to the presently observed VLBI radio core. Model building when combined with such observations ultimately may be capable of determining inner jet 
dynamics, e.g., the magnetic configuration and the acceleration region, but it will be a challenge to relate the observed appearance and spectrum to the dynamical flow structures and emission processes.

\section{Kiloparsec Scales: Entrainment, Structure, FRI/FRII, Ageing}

All extragalactic jets may be accelerated up to relativistic speeds, and observed galactic jets provide weak evidence that jets produced by black holes are relativistic (Mirabel \& Rodriguez 1995). Evidence for relativistic speeds on the kiloparsec scale is provided by observations of the proper motions of features in the M87 jet reviewed by John Biretta at the workshop. M87 jet features typically move at speeds of $0.5 \mathrm{c}$. That these typical speeds imply relativistic flow is suggested by modeling. For example, a model for knot $\mathrm{A}$ presented by Geoffrey Bicknell requires a Lorentz factor of $3-5$ to move a Kelvin-Helmholtz generated oblique shock at $0.5 \mathrm{c}$ along a jet with proper jet density less than the external density by a factor of $10-100$. Note however that scissors like effects arising from differentially moving multiple mode structures and/or projection of differentially moving near and far side jet structures can produce the observed (Biretta 1993) nearly stationary features and features with speeds up to about 3c. Overly simplistic interpretation of moving features must be avoided in the future.

While subluminal motion does not require sub-relativistic jet speeds, there are compelling reasons to believe that jets must slow down on kiloparsec scales. Slowdown occurs as a result of mixing and entrainment at the jet-external medium interface. As presented by David DeYoung a boundary layer develops in which large scale structures gulp material which is then digested at smaller scales in a non-linear and fully turbulent process. A quasi-steady state exists at the larger scales. Three dimensional MHD simulations presented by Philip Hardee reveal that light jets entrain and mix when the large scale Kelvin-Helmholtz modes develop significant amplitude, and the entrainment of more dense external material leads to relatively rapid slow down and disruption of ordered jet structure. Such interaction should lead to a sheath and spine jet structure such as that espoused by Robert Laing (1993) to explain many of the observed magnetic field and surface brightness structures along kiloparsec scale jets.

Three dimensional MHD simulations performed by David Clarke have revealed that the formation of emission filaments observed in radio lobes is caused by shearing within a turbulent lobe. Michael Norman presented a numerical simulation which showed that jets can remain relatively stable until significant lobe turbulence is encountered if no regular driving frequency such as that provided by jet precession is present. Overall the three dimensional numerical simulations imply that powerful jets protect 
themselves from serious entrainment effects by inflating a tenuous lobe or cocoon. These jets can remain well collimated until large scale distortions grow to large amplitudes and present results suggest that such jets could remain relativistic to large scales. Less powerful jets not protected by a tenuous lobe or cocoon should slow as they develop an extensive entraining boundary layer and lead to FRI radio sources (e.g., Bicknell 1986). Further evidence for a decelerated relativistic jet FRI radio source model was presented by Geoffrey Bicknell. Relatively simple assumptions relating a galaxy's core radius and pressure to the development of transonic entraining jet flow and to the radio luminosity resulted in an excellent fit to the slope and position of the dividing line in the radio-optical luminosity plane between FRI and FRII type radio sources (e.g., Owen \& Ledlow 1994).

The numerical simulations reveal shock structures and magnetic filamentation both within jets and in the lobes. Jean Eilek pointed out that these two features provide in situ acceleration sites and relatively loss free regions for the radiating electron population. These features can serve to explain the discrepancy between dynamically estimated source ages and conventionally determined spectral ageing source ages. However, Larry Rudnick provided compelling reasons to remain sceptical of conventional spectral ageing arguments. In any event, the effects of multiple weak shock particle acceleration sites and particle diffusion through magnetic filaments will need to be included in future modeling efforts.

\section{Jets on Cluster Scales}

The tenuous plasma in radio jets should prove susceptible to gradients in the external environment and perhaps can be used as probes of the external environment. However, as noted by Mark Birkinshaw, jets create their own environment. Still, the less powerful jets which do not create protective lobes should be more influenced by the environment. In particular, the distorted tailed radio sources must be shaped by interaction with the intracluster medium (ICM). Jagbir Hooda reported results from numerical simulation of the behavior of a three dimensional jet crossing an oblique ISM/ICM interface. Such an oblique interface appears incapable of distorting jets as appears to be necessary to form the Wide Angle Tailed (WAT) structures associated with dominant $(\mathrm{D}$ or $\mathrm{cD})$ galaxies that generally lie at the optical or X-ray centroids of clusters. However, Jack Burns noted that numerical simulations of cluster merger events (Röettiger, Burns \& Loken 1993) lead to transonic motions of the ICM relative to the optical centroid of the cluster, and that other simulations of jet propagation in crossflows (Loken et al. 1995) show that jets can be bent into the appropriate shapes. 


\section{Final Remarks}

Traditional theory combined with numerical simulations has shown real progress in the past decade. A synergy between theory, numerical simulation, and observation barely envisioned a decade ago has developed. This is particularly apparent in the combined theoretical and numerical studies of jet dynamics, inspired by the radio observations. In part, future development waits on computational and observational hardware. Still, the detailed multi-frequency observations that are now becoming available deserve detailed modeling and interpretive efforts. The major challenge that remains to any modeling and interpretive effort is to tie emission processes and microphysics to the magnetohydrodynamics.

\section{Acknowledgements}

P. Hardee thanks SOC members G. Bicknell, A. Bridle, J. Burns, R. Laing, A. Marscher, M. Norman, P. Scheuer, \& A. Zensus, and also A. Rosen, J. Wardle, \& P. Wiita. The NSF provided workshop support through EPSCoR grant OSR-9108761 and research support through grant AST-9318397.

\section{References}

Balbus, S.A. \& Hawley, J.F. (1991) A Powerful Local Shear Instability in Weakly Magnetized Disks I. Linear Analysis, Astrophysical Journal, 376, pp.214-222.

Bicknell, G.V. (1986) A Model for the Surface Brightness of a Turbulent Low Mach Number Jet. III. Adiabatic Jets of Arbitrary Density Ratio: Application to NGC 315, Astrophysical Journal, 305, pp.109-130.

Biretta, J.A. (1993) The M87 Jet, Astrophysical Jets, eds. D. Burgarella, M. Livio, \& C.P. O'Dea, Cambridge University Press, Cambridge, pp.263-304.

Birkinshaw, M. (1991) The Stability of Jets, Beams and Jets in Astrophysics, ed. P.A. Hughes, Cambridge University Press, Cambridge, pp.278-341.

Blandford, R. (1993) Acceleration and Collimation Mechanisms in Jets, Astrophysical Jets, eds. D. Burgarella, M. Livio, \& C.P. O'Dea, Cambridge University Press, Cambridge, pp.15-29.

Laing, R.A. (1993) Radio Observations of Jets: Large Scales, Astrophysical Jets, eds. D. Burgarella, M. Livio, \& C.P. O'Dea, Cambridge University Press, Cambridge, pp.95-119.

Loken, C., Röettiger, K., Burns, J., \& Norman, M. (1995) Radio Jet Propagation \& WideAngle Tailed Radio Sources in Merging Galaxy Cluster Environments, Astrophysical Journal, 445, pp.80-97.

Mirabel, I.F. \& Rodriguez, L.F. (1995) Superluminal Motions in our Galaxy, 17th Texas Symposium, Annals of the New York Academy of Sciences, in press.

Owen, F.N. \& Ledlow, M.J. (1994), The FR I/II Break and the Bivariate Luminosity Function in Abell Clusters of Galaxies, The First Stromlo Symposium: The Physics of Active Galaxies, eds. G.V. Bicknell, M.A. Dopita, \& J. Quin, ASP Conference Series

Röettiger, K., Burns, J., \& Loken, C. (1993) When Clusters Collide - A Numerical Hydro/N-body Simulation of Merging Galaxy Clusters, Astrophysical Journal Letters, 407, pp.53-56. 